\title{
Nach Krebs: lebenslang Probleme mit den Harnwegen
}

Überlebende von Krebs in der Kindheit werden im späteren Leben zweieinhalb mal häufiger als Vergleichspersonen wegen einer Erkrankung der Harnwege stationär behandelt. Das größte Risiko besteht nach Abdominaltumoren.

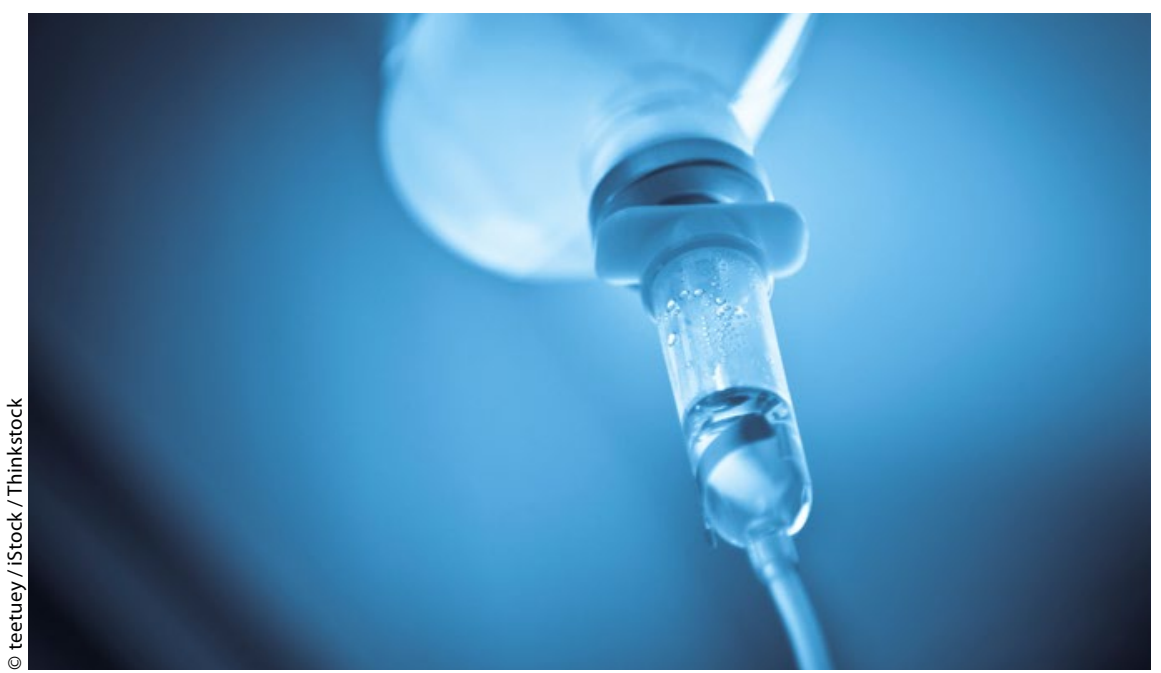

Das Risiko für Folgeerkrankungen ist vor allem für jugendliche Chemotherapiepatienten hoch.

n den nordischen Ländern überleben nach einer Krebserkrankung in der Kindheit heute mehr als $80 \%$ der Patienten die Fünfjahresgrenze. Fast jeder tausendste Erwachsene in der Allgemeinbevölkerung hat ein Malignom überstanden. Viele haben aber noch lange mit den Folgen der Therapien zu kämpfen.

Nur wenig ist bisher über die Auswirkungen von nephrotoxischen Chemotherapien, Nephrektomien und Strahlentherapien im Bereich des Abdomens auf den Harntrakt bekannt. Bei der Adult Life after Childhood Cancer in ScandinaviaStudie haben Trine Bonnesen von der Uniklinik Aarhus und Kollegen untersucht, wie häufig Überlebende kindlicher Malignome in Dänemark, Finnland, Island, Norwegen und Schweden solche Folgeerkrankungen des Harntrakts entwickeln. Hierzu analysierten sie die Daten von 43.909 Personen, die vor dem 20. Lebensjahr an Krebs erkrankt waren. Der Studienzeitraum reichte von den 1940er-Jahren bis Ende 2008. Am häu- figsten kamen Tumoren des ZNS, Leukämien und Lymphome vor. Zum Vergleich zogen die Autoren pro Patient in der Regel fünf vergleichbare Personen ohne Krebserkrankung heran. Ausgewertet wurden die Daten der nationalen Klinikregister, in denen 32.519 Ein-Jahres-Überlebende im Mittel weitere zehn Jahre nachbeobachtet worden waren.

Insgesamt waren 1.645 Überlebende wegen einer Erkrankung des Harntrakts stationär behandelt worden. Die Autoren errechneten für die ehemaligen Krebspatienten gegenüber den Kontrollen ein 2,5-faches Erkrankungsrisiko beziehungsweise ein absolutes Zusatzrisiko (AER) von 229 zusätzlichen Fällen pro 100.000 Personenjahre (PJ). Dieses Risiko war für alle untersuchten Krankheitskategorien erhöht. Diese waren glomeruläre Erkrankungen, tubulointerstitielle Krankheiten, akutes Nierenversagen, chronische Nierenkrankheiten, Urolithiasis, obstruktive Uropathie, Harnwegsinfektionen, andere und un- spezifische Erkrankungen. Am häufigsten wurde eine Infektion der Harnwege diagnostiziert. Das Gesamtrisiko für ein akutes Nierenversagen war gegenüber den Kontrollen siebenfach erhöht, das für eine chronische Nierenerkrankung vierfach. Die höchsten relativen Risiken im Vergleich zu den Kontrollen zeigten sich bei den medikamenteninduzierten tubulointerstitiellen Nierenerkrankungen (RR 15,6) sowie bei der neuromuskulären Dysfunktion der Blase (RR 11,1).

Abgesehen von den Überlebenden eines Retinoblastoms ergab sich in allen Krebsdiagnosegruppen ein erhöhtes $\mathrm{Ri}$ siko für Erkrankungen der Harnwege. Insgesamt am häufigsten mussten Überlebende eines Neuroblastoms oder eines Nierentumors wegen der Folgeerkrankung stationär behandelt werden. Das Risiko für ein akutes Nierenversagen war besonders hoch bei Patienten, die in der Kindheit an Neuroblastomen, Nierentumoren oder Leukämie erkrankt waren. Ehemalige Patienten mit Neuroblastom, Nieren- oder Lebertumor entwickelten zudem besonders oft eine chronische Nierenkrankheit. Anders als in früheren Studien war das Risiko für Folgeerkrankungen aber nicht bei denjenigen am größten, deren Krebs in besonders jungen Jahren diagnostiziert worden war, sondern vielmehr bei den zwischen 15 und 19 Jahren Erkrankten (HR 2,39 gegenüber den 0 - bis 4-Jährigen).

Fazit: Die Studie verdeutliche unter anderem, so die Autoren, wie lang die zusätzliche Belastung des Harntrakts nach einer Krebsdiagnose unvermindert bestehen bleibe und dass fast für alle Erkrankungen in diesem Bereich eine erhöhte Gefahr bestehe. Auffällig war unter anderem das besonders hohe Risiko für ein akutes Nierenversagen nach Leukämien, Neuroblastomen und Nierentumoren. Hier könnten neben nierentoxischen Chemotherapeutika auch zum Teil zeitgleiche längerfristige Antibiotikatherapien mit nephrotoxischen Substanzen wie Aminoglykosiden oder Vancomycin beziehungsweise hochdosiertem Methotrexat relevant sein. Dr. Christine Starostzik

Bonnesen TG et al. Long-term risk of renal and urinary tract diseases in childhood cancer survivors: A population-based cohort study. Euro J Can. 2016;64:52-61. 